\title{
Energy Minimization in Wireless Multihop Networks Using Two-Way Network Coding
}

\author{
Lianghui Ding*, Ping Wu*, Hao Wang ${ }^{\dagger *}$, Zhiwen Pan $^{\dagger}$ and Xiaohu You ${ }^{\dagger}$ \\ * Signals and Systems, Dept. of Engineering Sciences, Uppsala University, Uppsala, Sweden \\ ${ }^{\dagger}$ National Mobile Communication Research Laboratory, Southeast University, Nanjing, China \\ Emails: $\{$ lhding, ping.wu\}@angstrom.uu.se, \{hao_wang, pzw, xhyu $\}$ seu.edu.cn
}

\begin{abstract}
The total energy minimization in wireless multihop networks using two-way network coding is investigated in this paper. The problem is first formulated as a linear programming problem, then decomposed into two sub-problems using the Lagrangian decomposition, and finally solved with the subgradient method. After that, the backpressure based algorithm is proposed to solve the problem in a distributed manner. The performance of the algorithm is evaluated first in a simple topology for analysis and then in a random topology with different number of flows for practical consideration. Simulation results show that the convergence time increases as the number of nodes in the network, and the energy cost per packet can be saved up to $30 \%$ by using two-way network coding.
\end{abstract}

Index Terms-Energy Efficiency, Two-Way Network Coding, Backpressure, Wireless Multihop Networks

\section{INTRODUCTION}

The key idea of network coding (NC) is to give routers the data processing ability rather than pure forwarding. It has been proved that the multicast rate, i.e., the max-flow/min-cut from the source to all sink nodes, can be realized with NC [1], and much research has been done on various aspects of NC, such as coding approaches [2], network utility optimization with NC [3]-[5], and cost minimization with NC [6]-[8], etc. In general, NC can be classified into two categories: inter-session and intra-session NC. Since there are much more coexisting flows in a practical network, and even simple NC approaches can obtain significant performance gain [9], construction of efficient and easily-applied inter-session $\mathrm{NC}$ is more attractive and valuable for practical networks.

Since the number of transmissions, and thus the energy cost, can be reduced by using NC, energy efficient $\mathrm{NC}$ has received much attention recently. Energy efficient multicast with NC has been theoretically analyzed in [6] and solved using distributed subgradient method in [8]. An energy efficient opportunistic network coding, COPR, was proposed for intersession $\mathrm{NC}$ in [7]. However, $\mathrm{NC}$ is done in an opportunistic way rather than being optimized in [7].

In this paper, we incorporate NC into optimization framework and jointly consider energy minimization, routing, scheduling, and inter-session NC. Since NC using overhearing includes at least 3 nodes, which causes extra overhead of complicated cooperation between neighboring nodes in network scheduling and need more buffer space, we only consider twoway $\mathrm{NC}$ in this paper. We formulate the problem as a linear programming problem, solve it using the dual subgradient method, and then propose the distributed backpressure algorithm to realize the solution in a distributed manner. Finally we evaluate the performance via simulation and show that twoway NC can save up to $30 \%$ of the energy cost.

The rest of the paper is organized as follows. We first introduce related work in Section II, and give some preliminary introduction in Section III. Then we discuss the problem formulation, dual decomposition, and the solution in Section IV. Afterwards, we propose the distributed backpressure algorithm in Section V, evaluate the performance in Section VI, and conclude the whole paper in Section VII.

\section{RELATED WORK}

Although optimal inter-session network coding is still an open question, a few efforts have been made on application of inter-session NC. It has been investigated using poisoningantidote method and linear programming in [11]. Opportunistic NC is implemented in an IEEE 802.11-based wireless ad hoc network [9]. Pairwise inter-session NC, which includes both butterfly and XOR with two sessions as special cases, has been proposed in [2] and optimized in a random network in [4].

As for network optimization with inter-session NC, joint scheduling and $\mathrm{NC}$ is analyzed and a backpressure-based algorithm is proposed in [14]. Joint routing and NC has been analyzed theoretically using linear programming in [3]. Energy cost minimization using cross-layer optimization with $\mathrm{NC}$ is investigated in [7]. However, only energy minimization by using $\mathrm{NC}$ is formulated in [7] without considering codingaware routing. Joint optimization of network utility and pairwise inter-session NC (PINC) [2] is formulated and solved in [4].

\section{PRELIMINARIES}

\section{A. Network Model}

A static wireless multihop network is modeled as a directed graph $G=\{V, E\}$, where $V$ is the set of nodes, $E$ is the set of links. A link $\{i, j\} \in E$ denotes that there exists a connection between nodes $i$ and $j$, i.e., the transmission of node $i$ can be correctly received by $j$ with probability almost equal to 1 . $N(i)$ is used to denote the set of neighboring nodes at $i$. We assume that the erasure wireless channel can be masked by adaptive modulation, channel coding, and retransmission, etc., and the rate $r_{i j}$ is fixed when link $(i, j)$ is scheduled. A unicast flow is defined as the data transfer from a source node to a 
destination node. Throughout the whole paper, we use $c$ and $\mathcal{C}$ to denote a specific commodity and the set of all commodities, respectively; $s^{c}$ and $d^{c}$ denote the source and destination nodes of commodity $c$, respectively.

\section{B. Network Coding}

In this paper, we limit our analysis to two-way inter-session $\mathrm{NC}$, and use linear XOR as the packet operation. As shown in Figure 1 , nodes $x$ and $z$ exchange data through node $y$. Node $y$ does XOR NC on received packets $P_{x}, P_{z}$ and multicasts $P_{x} \oplus P_{z}$ to nodes $x$ and $z$. Then nodes $x$ and $z$ can obtain packets $P_{z}, P_{x}$ through decoding $P_{x} \oplus P_{z}$ with existed packets $P_{x}, P_{z}$. The lengths of all packets are assumed to be equal.

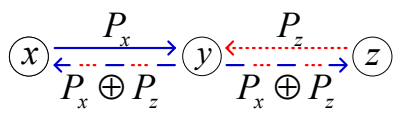

Fig. 1. Two-way network coding.

We use $\left(c, c^{\prime}\right)$ and $M=\left\{\left(c, c^{\prime}\right)\right\}$ to denote a possible twoway NC scheme and the set of all possible coding schemes, respectively. For uncoded transmission, we use $f_{i j}^{c}$ to denote the transmission rate from nodes $i$ to $j$ of commodity $c$. For two-way $\mathrm{NC}$, let $f_{(i,(j, k))}^{c, c^{\prime}}$ denote the coded transmission rate from nodes $i$ to $j$ of commodity $c$, and $f_{(i,(k, j))}^{c^{\prime}, c}$ denote the coded transmission from $i$ to $k$ of commodity $c^{\prime}$. Note that $j, k$ are previous hop nodes of commodity $c^{\prime}, c$, respectively, and $f_{(i,(j, k))}^{c, c^{\prime}}=f_{(i,(k, j))}^{c^{\prime}, c}$. For notation convenience, we use $f_{(i, j, k)}^{c, c^{\prime}}$ to denote the coded transmission rate from $i$ to $j$ and $k$ of commodities $c$ and $c^{\prime}$.

We use $P_{(i, j)}, P_{(i, j, k)}=\max \left(P_{(i, j)}, P_{(i, k)}\right)$ to denote the transmission power cost of $f_{(i, j)}^{c}$ and $f_{(i, j, k)}^{c, c^{\prime}}$, where $P_{(i, j)}$ is the transmission power cost of the link $(i, j)$. Since the receiving power is often much lower than that for transmission, it is not considered in this paper.

\section{Achievable Rate Region}

Let $\pi, \Pi$ denote a scenario of scheduling and the set of all scheduling scenarios, the achievable rate region of the network with time-sharing can be written as

$$
\begin{array}{r}
C o(\Pi)=\left\{\left(r_{i j}, r_{(i, j, k)}\right): \sum_{\pi \in \Pi} \alpha_{\pi}\left(r_{i j}^{\pi}, r_{(i, j, k)}^{\pi}\right),\right. \\
\left.\alpha_{\pi} \geq 0, \sum_{\pi \in \Pi} \alpha_{\pi}=1\right\}
\end{array}
$$

where $\alpha_{\pi}$ is the time shared by scheduling $\pi, r_{i j}^{\pi}, r_{(i, j, k)}^{\pi}$ are the uncoded transmission rate of link $(i, j)$, and coded transmission rate of $(i, j, k)$ in $\pi$, respectively. $\operatorname{Co}(\Pi)$ is the convex hull of all achievable rate set $\left(r_{i j}, r_{(i, j, k)}\right)$.

\section{Problem Formulation And Solution}

\section{A. Problem formulation}

According to system models introduced in Section III, we can formulate the problem as

$$
\begin{gathered}
\min \sum_{i \in N}\left(\sum_{c \in \mathcal{C}} \sum_{(i, j) \in E} f_{i j}^{c} P_{i j}+\sum_{(i, j, k)} \sum_{\left(c, c^{\prime}\right)} f_{(i, j, k)}^{c, c^{\prime}} P_{(i, j, k)} / 2\right) \\
\text { s.t. } f_{j i}^{c}+\sum_{\left(c, c^{\prime}\right)} \sum_{\substack{k \in N \\
k \neq j}} f_{(j,(i, k))}^{c, c^{\prime}}=g_{j i}^{c}+\sum_{\substack {\left(c, c^{\prime}\right) \\
\begin{subarray}{c}{k \in N \\
k \neq j{ ( c , c ^ { \prime } ) \\
\begin{subarray} { c } { k \in N \\
k \neq j } }\end{subarray}} f_{(i,(k, j))}^{c, c^{\prime}}, \forall(j, i), c \\
\sigma_{i}^{c}+\sum_{(j, i) \in E} g_{j i}^{c}=\sum_{(i, j) \in E} f_{i j}^{c}, \forall i, c \\
\sum_{c \in \mathcal{C}} f_{i j}^{c} \leq r_{i j}, \forall(i, j) \\
\sum_{\left(c, c^{\prime}\right)} f_{(i, j, k)}^{c, c^{\prime}} \leq r_{(i, j, k)} \forall(i, j, k) \\
\left(r_{i j}, r_{(i, j, k)}\right) \in C o(r) \\
f_{i j}^{c} \geq 0, f_{(i, j, k)}^{c, c^{\prime}} \geq 0, g_{j i}^{c} \geq 0, \forall i, j, k, c, c^{\prime}
\end{gathered}
$$

The objective function is the total power cost for transfer of all commodities in $\mathcal{C}$. Constraints in (3) (4) are flow conservation laws. Here we use $g_{j i}^{c}$ to denote the virtual unicast output data rate of data from link $(j, i)$ [5] for easier implementation of subgradient and backpressure algorithms. All data of commodity $c$ from link $(j, i)$ is either transmitted in multicast over coded links such as $(i, j, k)$ or in unicast with virtual rate $g_{j i}^{c}$. Note that the virtual unicast data transmission does not cost any power. Equation (3) shows that the total output rates of commodity $c$ at node $i$ in terms of link $(j, i)$ are equal to the corresponding input rates from link $(j, i)$. Equation (4) depicts that the total unicast output rates of commodity $c$ at node $i$ are equal to the sum of all input unicast transmission demands. The source rate $\sigma_{i}^{c}$ is defined as

$$
\sigma_{i}^{c}=\left\{\begin{aligned}
x^{c}, & \text { if } i=s^{c}, \\
-x^{c}, & \text { if } i=d^{c}, \\
0, & \text { otherwise. }
\end{aligned}\right.
$$

where $x^{c}$ is the traffic demand of the commodity $c$.

Equations (5) (6) are the physical rate constraints, i.e., the transmission rates over links $(i, j)$ and $(i, j, k)$ should be less than the scheduled rates. All scheduled rates are bounded by the achievable rate region $C o(\Pi)$ as shown in (7).

Equation (8) is the boundary conditions of all variables.

\section{B. Lagrange Dual Decomposition}

By introducing Lagrange multipliers $\lambda_{j i}^{c}$ and $\gamma_{i}^{c}$ to relax each constraint in (3) and (4), respectively, the corresponding partial Lagrangian function can be written as $L\left(\mathbf{f}, \mathbf{f}_{\mathbf{n c}} ; \boldsymbol{\Lambda}, \boldsymbol{\Gamma}\right)$, where $\mathbf{f}, \mathbf{f}_{\mathbf{n c}}$ represent the primal variable vectors of uncoded flow rates and coded flow rates, respectively; $\boldsymbol{\Lambda}$ and $\boldsymbol{\Gamma}$ represent the dual variable vectors $\lambda_{j i}^{c}$ and $\gamma_{i}^{c}$. With this Lagrangian function, we can write the dual objective function as

$$
g(\boldsymbol{\Lambda}, \boldsymbol{\Gamma})=\inf _{\mathbf{f}, \mathbf{f}_{\mathbf{n c}}} L\left(\mathbf{f}, \mathbf{f}_{\mathbf{n c}} ; \boldsymbol{\Lambda}, \boldsymbol{\Gamma}\right)
$$


We first note that all traffic demands have to be satisfied by the network with $\mathrm{NC}$, otherwise energy minimized routing does not exist. Henceforth, we can assume that there always exist routing paths with NC for all flows, i.e., (5) and (6) are strictly satisfied. With this assumption, the Slater's condition for constraint qualification will also be fulfilled [15]. In addition, since the objective function of the primal problem is linear, strong duality holds [15], and we can solve the primal problem through the dual function

$$
\max g(\boldsymbol{\Lambda}, \boldsymbol{\Gamma})
$$

Next we consider how to get the primal variables $\mathbf{f}^{(\mathbf{k})}, \mathbf{f}_{\mathbf{n c}}^{(\mathbf{k})}$ at the point $\left(\boldsymbol{\Lambda}^{(\mathbf{k})}, \boldsymbol{\Gamma}^{(\mathbf{k})}\right)$ with dual decomposition. With some transformation, the dual function can be written as

$$
g(\boldsymbol{\Lambda}, \boldsymbol{\Gamma})=-\sum_{i \in N} \sum_{c \in \mathcal{C}} \gamma_{i}^{c} \sigma_{i}^{c}-\max \Phi-\max \Psi
$$

where,

$$
\begin{gathered}
\Phi=\sum_{i \in N} \sum_{(j, i) \in E} \sum_{c \in \mathcal{C}} g_{j i}^{c}\left(\lambda_{j i}^{c}-\gamma_{i}^{c}\right) \\
\Psi=\sum_{i \in N} \sum_{(i, j) \in E} \sum_{c \in \mathcal{C}} f_{i j}^{c} y_{i j}^{c}+\sum_{i \in N} \sum_{(i, j, k)} \sum_{\left(c, c^{\prime}\right)} f_{(i, j, k)}^{c, c^{\prime}} z_{(i, j, k)}^{c, c^{\prime}}
\end{gathered}
$$

and

$$
\begin{gathered}
y_{i j}^{c}=\gamma_{i}^{c}-\lambda_{i j}^{c}-P_{i j} \\
z_{(i, j, k)}^{c, c^{\prime}}=\left(\lambda_{j i}^{c}-\lambda_{i k}^{c}\right)+\left(\lambda_{k i}^{c^{\prime}}-\lambda_{i j}^{c^{\prime}}\right)-P_{(i, j, k)}
\end{gathered}
$$

According to Equation (12), the dual function is equivalent to solve the two subproblems: $\max \Phi$, and $\max \Psi$.

It is obvious that $\sum_{(j, i) \in E} g_{j i}^{c}$ is limited by the total input rates from all nodes $j \in N(i)$ to $i$, and let it be $V_{i}^{c}=\sum_{j \in N(i)} c_{j i}$. Since $g_{j i}^{c} \geq 0$, the solution of $\max \Phi$ will be attained at the extreme points, i.e.,

$$
g_{j i}^{c}= \begin{cases}V_{i}^{c}, & \text { if } \lambda_{j * i}^{c}-\gamma_{i}^{c}>0, \\ 0, & \text { otherwise. }\end{cases}
$$

where $j^{*}=\arg \max _{(j, i) \in E}\left(\lambda_{j i}^{c}-\gamma_{i}^{c}\right)$.

Similarly, according to the constraint $\sum_{c \in \mathcal{C}} f_{i j}^{c} \leq r_{i j}$, and $f_{i j}^{c} \geq 0$, we can obtain the solution of $\max \sum_{c \in \mathcal{C}} f_{i j}^{c} y_{i j}^{c}$ on the link $(i, j)$ as

$$
f_{i j}^{c}= \begin{cases}r_{i j}, & \text { if } c=c^{*} \text { and } y_{j i}^{c^{*}}>0 \\ 0, & \text { otherwise. }\end{cases}
$$

where $c^{*}=\arg \max _{c \in \mathcal{C}} y_{j i}^{c}$.

Considering the constraint $\sum_{\left(c, c^{\prime}\right)} f_{(i, j, k)}^{c, c^{\prime}} \leq r_{(i, j, k)}$, and $f_{(i, j, k)}^{c, c^{\prime}} \geq 0$, the solutions of $\max \sum_{\left(c, c^{\prime}\right)} f_{(i, j, k)}^{c, c^{\prime}} z_{(i, j, k)}^{c, c^{\prime}}$ on the coded link $(i, j, k)$ can be written as

$$
f_{(i, j, k)}^{c, c^{\prime}}= \begin{cases}r_{(i, j, k)}, & \text { if }\left(c, c^{\prime}\right)=\left(c^{*}, c^{\prime *}\right) \text { and } z_{(i, j, k)}^{c^{*}, c^{\prime *}}>0 \\ 0, & \text { otherwise. }\end{cases}
$$

where $\left(c^{*}, c^{*}\right)=\arg \max _{\left(c, c^{\prime}\right)} z_{(i, j, k)}^{c, c^{\prime}}$.

Thus, the dual problem is transformed to

$$
\max \sum_{i \in N}\left[\sum_{(i, j) \in E} r_{i j}\left(y_{i j}^{c^{*}}\right)^{+}+\sum_{(i, j, k)} r_{(i, j, k)}\left(z_{(i, j, k)}^{c^{*}, c^{\prime *}}\right)^{+}\right]
$$

which is to select the scheduled transmission link sets so as to maximize the weighted sum of all links. It depends on the interference model of the wireless network and is generally NP-hard. When the the primary interference model is used, the problem is equivalent to weighted hypergraph matching and can be approximated in a distributed manner [13].

\section{Subgradient Algorithm}

Let primal variables $\mathbf{f}^{(\mathbf{k})}, \mathbf{f}_{\mathbf{n c}}^{(\mathbf{k})}$ denote the solution of the dual problem obtained at the point $\Lambda^{\mathbf{c}(\mathbf{k})}, \Gamma^{\mathbf{c}(\mathbf{k})}$, i.e.,

$$
\left(\mathbf{f}^{(\mathbf{k})}, \mathbf{f}_{\mathbf{n c}}^{(\mathbf{k})}\right)=\max g\left(\boldsymbol{\Lambda}^{\mathbf{c}(\mathbf{k})}, \boldsymbol{\Gamma}^{\mathbf{c}(\mathbf{k})}\right)
$$

Since $g(\boldsymbol{\Lambda}, \boldsymbol{\Gamma})$ is a concave function, we consider its supergradients. Given the primary solution $\mathbf{f}^{(\mathbf{k})}, \mathbf{f}_{\mathbf{n c}}^{(\mathbf{k})}$, the supergradients of $g(\boldsymbol{\Lambda}, \boldsymbol{\Gamma})$ are

$$
\begin{gathered}
h\left(\lambda_{j i}^{c}\right)^{(k)}=f_{j i}^{c}+\sum_{\left(c, c^{\prime}\right)} \sum_{k \in N, k \neq j}\left(f_{(j,(i, k))}^{c, c^{\prime}}-f_{(i,(k, j))}^{c, c^{\prime}}\right)-g_{j i}^{c} \\
h\left(\gamma_{i}^{c}\right)^{(k)}=\sigma_{i}^{c}+\sum_{(j, i) \in E} g_{j i}^{c}-\sum_{(i, j) \in E} f_{i j}^{c}
\end{gathered}
$$

where $f_{i j}^{c}, f_{(j,(i, k))}^{c, c^{\prime}}$ are elements of primal variable vectors $\mathbf{f}^{\mathbf{k}}, \mathbf{f}_{\mathbf{n c}}^{\mathbf{k}}$, respectively.

Then, starting from an initial point $\Lambda^{(\mathbf{0})}, \Gamma^{(\mathbf{0})}$, we can do following iteration to converge to the optimal solution.

$$
\begin{aligned}
& \lambda_{i}^{c(k+1)}=\left[\lambda_{i}^{c(k)}+\alpha_{k} h\left(\lambda_{j i}^{c}\right)^{(k)}\right]^{+} \\
& \gamma_{i j}^{c(k+1)}=\left[\gamma_{i j}^{c(k)}+\alpha_{k} h\left(\gamma_{i}^{c}\right)^{(k)}\right]^{+}
\end{aligned}
$$

where $\alpha_{k}$ is the step size for iteration, $[x]^{+}=\max (x, 0)$.

According to [15], subgradient method is guaranteed to converge to the optimum of the convex problem if $\alpha_{k}$ satisfies

$$
\alpha_{k} \rightarrow 0, \quad \sum_{k=1}^{\infty} \alpha_{k}=\infty
$$

\section{BACK Pressure Algorithm}

The traditional backpressure algorithm can not be applied here directly since the two-way NC does not only depend on the next hop node, but also on the previous hop node as shown in Section IV. For a successful transmission of a packet with $\mathrm{NC}$, we have to consider which node it comes from. Therefore, we establish a new queue structure for the two-way NC here. We allocate $|N(i)||\mathcal{C}|$ broadcast buffers for all commodities from all neighboring node, and $|\mathcal{C}|$ unicast buffers for all commodities, where $\left|N_{i}\right|,|\mathcal{C}|$ is the number of neighboring nodes of $i$, and the number of commodities, respectively. The data in unicast buffer of commodity $c$ at node $i$ has two input ways: one is from broadcast buffers through the virtual unicast transmission, and another is from upper application layer if $i$ is the source node of commodity $c$. For potential need of packet decoding, we further allocate $|N(i)||\mathcal{C}|$ side buffers for all transmitted packets received from node $j$. We use $B_{j i}^{c}, B_{i}^{c}$ and $S_{j i}^{c}$ to denote the broadcast buffer from node $j$, unicast buffer, and the side buffer from $j$ of commodity $c$ at node $i$, respectively. $Q_{j i}^{c(k)}$ and $Q_{i}^{c(k)}$ denote 
the buffer sizes of commodity $c$ from node $j$, and the unicast buffer size of commodity $c$ at time slot $k$, respectively.

Now let's consider the variance of queue sizes when a unicast link $(i, j)$ for $c$ or a multicast link $(i, j, k)$ for $\left(c, c^{\prime}\right)$ are scheduled with rate $\mathbf{f}^{(\mathbf{k})}, \mathbf{f}_{\mathbf{n c}}^{(\mathbf{k})}$. They are written as

$$
\begin{gathered}
Q_{j i}^{c(k+1)}=Q_{i}^{c(k)}+h\left(\lambda_{i j}^{c}\right)^{(k)} \\
Q_{c}^{(k+1)}=Q_{c}^{c(k)}+h\left(\gamma_{i}^{c}\right)^{(k)}
\end{gathered}
$$

where $h\left(\lambda_{i j}^{c}\right)^{(k)}, h\left(\gamma_{i}^{c}\right)^{(k)}$ are supergradients given in (22) and (23).

Comparing (27) and (28) with (24) and (25), we can find the relationship between queue sizes and dual variables as

$$
Q_{j i}^{c(k)}=\lambda_{j i}^{c(k)} / \alpha_{k}, \quad Q_{i}^{c(k)}=\gamma_{i}^{c(k)} / \alpha_{k}
$$

Thus we can use the distributed backpressure algorithm to solve the problem of energy minimized two-way NC. The details of each iteration are shown in Algorithm 1.

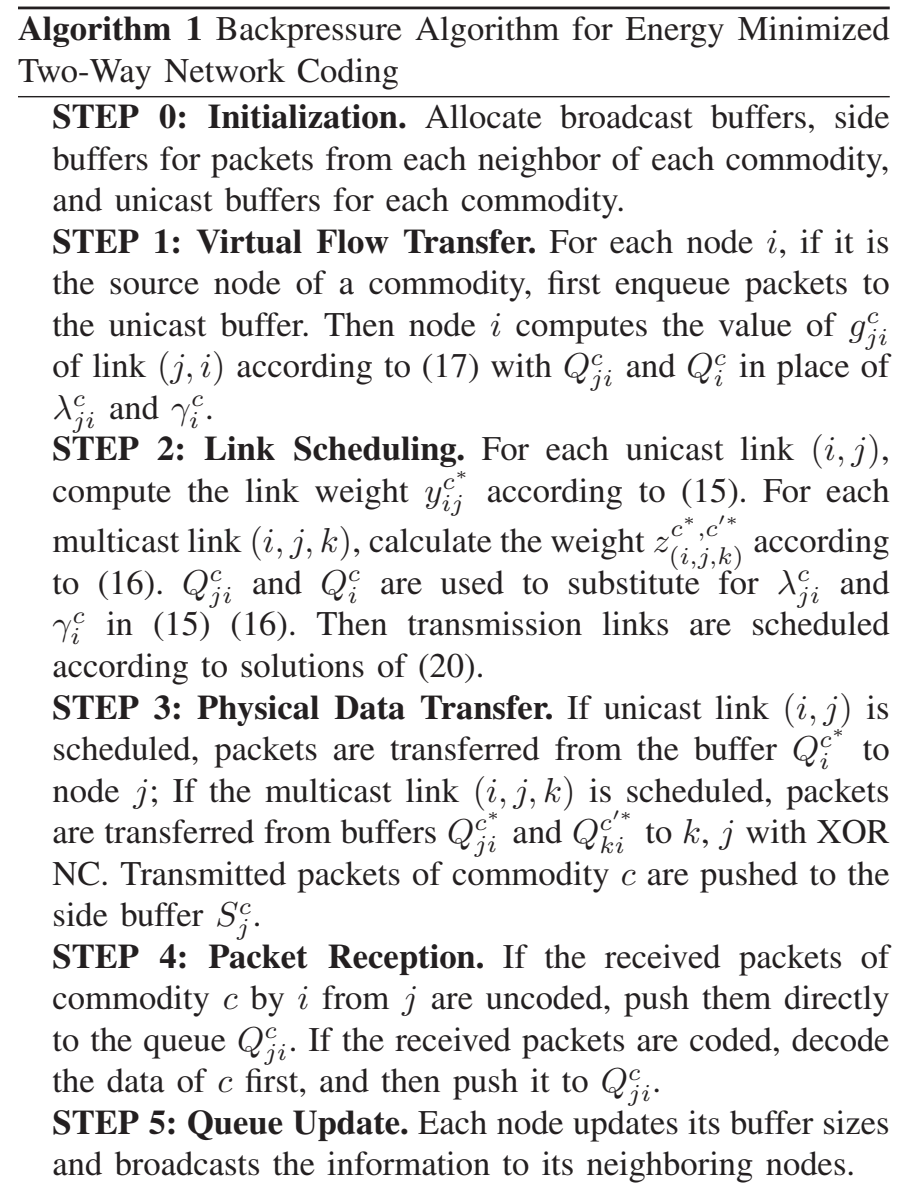

\section{Vi. Performance Evaluation}

In this section, we first show the performance of backpressure algorithm for energy minimized two-way $\mathrm{NC}$ in a simple topology for analysis, and then give the performance comparison between scenarios in a random topology for practical consideration.

\section{A. Simple Topology}

The simple topology is shown in Figure 2. We assume all links are symmetric, the rate of each link is $3 \mathrm{Kbps}$, and the packet size is $1 \mathrm{~K}$ bits. The energy cost of each link is labeled in Figure 2, e.g., that of link $(1,2)$ is 2 Jouls per second.

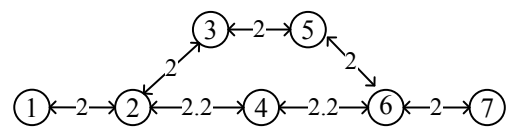

Fig. 2. Simple topology with 7 nodes.

Two flows with traffic demands $1 \mathrm{Kbps}$ are placed in this topology, one of which is from nodes 7 to 3 , and the other is from nodes 1 to 7 . The variance of energy cost per packet with the number of iterations is shown in Figure 3. After about 1000 iterations, the energy costs per packet with and without $\mathrm{NC}$ converge to 2.0 and 2.4. The energy cost with $\mathrm{NC}$ is about $20 \%$ lower than that without NC. Without NC, the flow from 1 to 7 is transmitted along the path $1 \rightarrow 2 \rightarrow 4 \rightarrow 6 \rightarrow 7$ with less energy cost. When two-way NC is used, the flow is redirected to the path $1 \rightarrow 2 \rightarrow 3 \rightarrow 5 \rightarrow 6 \rightarrow 7$ for the NC opportunities at nodes 5 and 6 . In both scenarios, the flow from nodes 7 to 3 is transferred through the path $7 \rightarrow 6 \rightarrow 5 \rightarrow 3$.

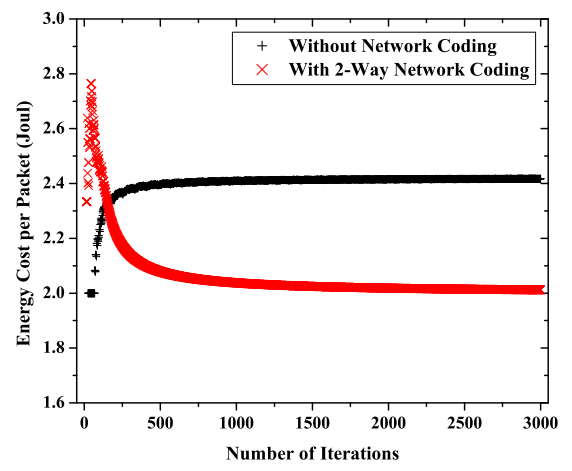

Fig. 3. Energy cost per packet in the simple topology.

\section{B. Random Topology}

Different from the appointed energy cost in the simple topology, here we utilize a more practical energy cost model as in [16]. The energy consumption per bit transmitted from node $i$ to $j$ is related to the distance between, i.e.,

$$
e_{i j}=e^{T}+\epsilon d_{i j}^{4}
$$

where $e^{T}=50 \mathrm{~nJ} / \mathrm{bit}$ is the constant energy cost of the transceiver, $\epsilon=0.1 \mathrm{~nJ} / \mathrm{bit} / \mathrm{m}^{4}$ is the energy cost of one bit transmission for one meter [16], and $d_{i j}$ is the distance between nodes $i$ and $j$. For correct reception of packets in the physical layer, we assume that the transmission range of each node is fixed at 15 meters, and all nodes in the transmission range can correctly decode the data without interference.

The random topology is shown in Figure 4. 20 nodes are randomly located in a $50 \mathrm{~m} \times 50 \mathrm{~m}$ square area, and two nodes in the transmission range of each other are connected with a 


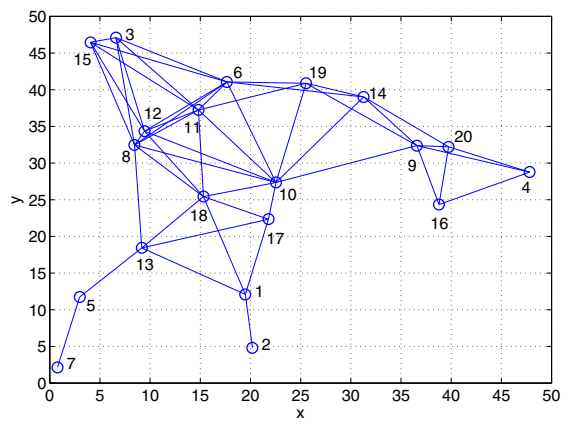

Fig. 4. Random topology with 20 nodes in the $50 \mathrm{~m} \times 50 \mathrm{~m}$ area.

solid line. We assume the rate of each link is $10 \mathrm{Kbps}$, and the packet size is $1 \mathrm{~K}$ bits.

We first analyze the performance of the proposed algorithm in a scenario with only two flows, which are from nodes 4 to 3 and from 12 to 9 with traffic demands equal to $1 \mathrm{Kbps}$. The variance of energy cost per packet with and without NC is shown in Figure 5. We can find that, in a random network with more nodes than that in the simple topology, the convergence rates of both are much slower. However, the convergence rate with $\mathrm{NC}$ is further slower than that without $\mathrm{NC}$, since there are more buffers to "push" in the algorithm with NC.

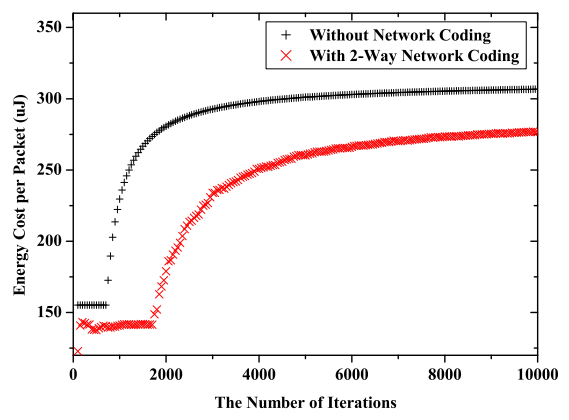

Fig. 5. Convergence of energy cost per packet in the random topology.

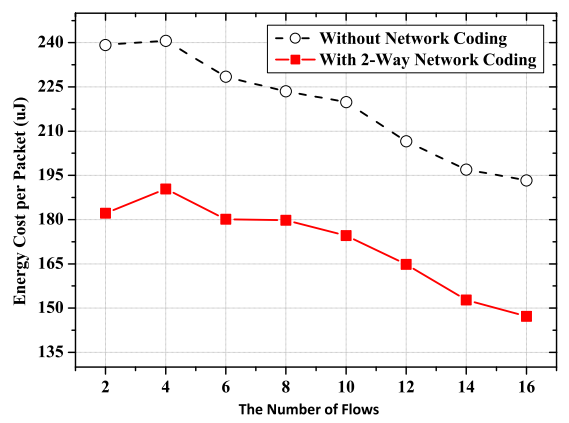

Fig. 6. Energy cost per packet according to the number of flows.

Next we consider the performance of the proposed backpressure algorithm with different number of flows in the aforementioned random topology. We randomly pick up two nodes as source and destination nodes of each flow, and run simulation for 100 times. After 10000 backpressure iterations, the final average energy costs per packet are shown in Figure 6. We can find that the average improvement ratio of energy cost per packet with NC is about $30 \%$ and does not change with the number of flows, although the energy cost per packet decreases with the number of flows.

\section{CONCLUSION}

In this paper, we have investigated the problem of energy minimization in wireless multihop networks using two-way NC. We formulate the problem as a linear programming problem and solve it by using the backpressure algorithm in a distributed manner. The performance of backpressure algorithm is evaluated via simulation in both simple and random topologies. Simulation results show that the energy cost can be saved up to $30 \%$ with two-way NC.

\section{REFERENCES}

[1] R. Ahlswede, N. Cai, S.-Y. R. Li, and R. W. Yeung, "Network information flow," IEEE Trans. on Information Theory, vol. 46, no. 4, pp. 1204-1216, Jul. 2000.

[2] C.-C. Wang and N. B. Shroff, "Pairwise intersession network coding on directed networks," To appear in IEEE Tran. on Information Theory, vol. 56, pp. 3879-3900, Aug. 2010.

[3] S. Sengupta, S. Rayanchu, and S. Banerjee, "An analysis of wireless network coding for unicast sessions: The case for coding-aware routing," in IEEE Proc. INFOCOM, May 2007.

[4] A. Khreishah, C.-C. Wang, and N. B. Shroff, "Cross-layer optimization for wireless multihop networks with pairwise intersession network coding," IEEE Journals on Selected Areas in Communications, vol. 27, pp. 606-621, Jun. 2009.

[5] L. You, P. Wu, Z. Pan, H. Hu, M. Song, and J. Song, "Cross-layer optimization of wireless multi-hop networks with network coding," in Proc. WCSP, Nanjing, China, Nov. 2009.

[6] Y. Wu, P. A. Chou, and S.-Y. Kung, "Minimum-energy multicast in mobile ad hoc networks using network coding," IEEE Trans. on Comm., vol. 53, no. 11, pp. 1906-1918, Nov. 2005.

[7] T. Cui, L. Chen, and T. Ho, "Energy efficient opportunistic network coding for wireless networks," in INFOCOM, Phoenix, AZ, USA, Apr 2008, pp. 361-365.

[8] D. S. Lun, N. Ratnakar, M. Medard, R. Koetter, et al, "Minimumcost multicast over coded packet networks," IEEE Tran. on Information Theory, vol. 52, no. 6, pp. 2608-2623, 2006.

[9] S. Katti, H. Rahul, W. Hu, D. Katabi, M. Medard, and J. Crowcroft, "XORs in the air: Practical wireless network coding," IEEE/ACM Trans. on Networking, vol. 16, no. 3, pp. 497-510, Jun. 2008.

[10] R. Dougherty, C. Freiling, and K. Zeger, "Insufficiency of linear coding in network information flow," IEEE Trans. on Information Theory, vol. 51, no. 8, pp. 2745-2759, Aug. 2005.

[11] D. Traskov, N. Ratnakar, D. S. Lun, etc., "Network coding for multiple unicasts: An approach based on linear optimization," in Proc. IEEE ISIT, Seattle, USA, Jul. 2006.

[12] T. Ho, and H. Viswanathan, "Dynamic algorithms for multicast with intra-session network coding," IEEE Trans. on Information Theory, vol. 55, no. 4, pp. 797-815, Feb 2009.

[13] T. Cui, L. Chen, and T. Ho, "Distributed optimization in wireless networks using broadcast advantage," in 46th IEEE Conference on Decision and Control, New Orleans, LA, USA, Dec 2007.

[14] P. Chaporkar, and A. Proutiere, "Adaptive network coding and scheduling for maximizing throughput in wireless networks," in MobiCom, Québec, Canada, Sep. 2007.

[15] S. Boyd, L. Vandenberghe, Covex Optimization. Cambridge University Press, 2004.

[16] J.-Hoo, Chang, and L. Tassiulas, "Maximum lifetime routing in wireless sensor networks," IEEE/ACM Trans. Networking, vol. 12, no. 4, pp. 609619, Aug. 2004. 